\title{
Inside the outbreaks
}

\section{The elite medical detectives of the epidemic intelligence service}

\author{
Mark Pendergrast \\ Houghton Mifflin Harcourt. Boston, Massachusetts, USA. 2010. \\ 432 pp. \$28.00. ISBN: 978-0-151-01120-9 (hardcover).
}

Reviewed by Vincent Racaniello

Columbia University Medical Center, New York, New York, USA.

E-mail: vrr1@columbia.edu

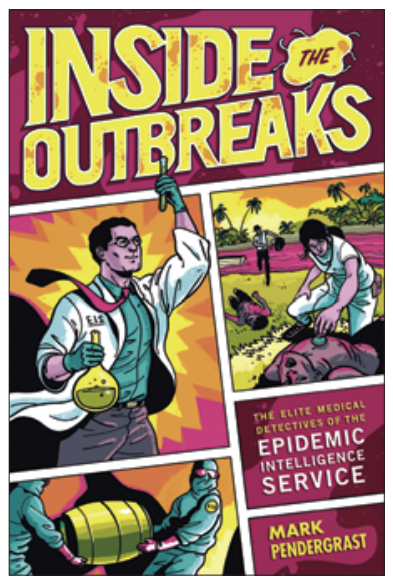

If there's something strange in your neigh-

borhood, who you gonna call? EIS!

In the early 1950s, Alexander Langmuir, an epidemiologist for the Communicable Disease Center (CDC) in Atlanta, Georgia, warned that pathogenic microbes could be used as agents of biological warfare. To counter the threat, he advised the federal government to establish a ready response team at CDC. This advice was prescient: when Korean hemorrhagic fever virus infected 25,000 American troops in June 1951, killing 3,000, funding was provided to establish the Epidemic Intelligence Service (EIS). The two-year program trained young epidemiologists not only to look out for biological warfare, but to respond quickly to unintentional epidemics.

Despite the success of EIS in producing the world's disease detectives, the history of the organization has never been told. Neither does Mark Pendergrast tell the history of EIS in Inside the Outbreaks - although it is a compelling collection of dozens of vignettes that cover many of the most interesting disease outbreaks of the past 60 years. If you are a microbe geek like I am, you will love reading about how EIS officers travel the world to quell lethal threats to global health.

All of the well-known infectious disease stories are here: pandemic influenza, the eradication of smallpox, the "Cutter incident" involving contaminated polio vaccine, and the first outbreak of Legionnaires' disease in Philadelphia, to name just a few. But there are many other less well-known incidents that established disease etiologies. An example is the finding by the EIS in 1955 of the importance of Staphylococcus aureus in hospital-acquired infections.
Inside the Outbreaks is divided into three sections: "The Grand Adventures of Dr. Langmuir's Boys" covers 1951-1970; “The Golden Age of Epi” continues to 1982; and "Complex Challenges" takes us to the present. Each section is composed of individual chapters that are further broken down into outbreak stories, such as "Mystery in Tuba City," "Profuse Diaphoresis in Infants," and "An Exhausting Disease." While I found this approach appealing, it does have weak points. Because of the focus on outbreaks, there is no overall view of the history of the EIS. Furthermore, character development is minimal: there are few memorable individuals, with the exception of Dr. Langmuir. This book is about outbreaks, not people. While EIS officers obviously play important roles in each story, we quickly forget them as we move on to the next problem.

There are so many riveting stories in Inside the Outbreaks that I had difficulty identifying one that conveyed the book's atmosphere. One of my favorites is "Health-Conscious Sprout Eaters," which describes outbreaks with Salmonella or E. coli $\mathrm{O} 157: \mathrm{H} 7$ caused by alfalfa sprouts. The sprouts, consumed uncooked, are difficult to sterilize because the bacteria may be internalized in the inner plant tissues. Sprouts contaminated with $E$. coli O157:H7 were tracked to Idaho farms, where deer droppings may have been the source of the bacteria. EIS officer Roger Shapiro concluded, "Raw sprouts are inherently dangerous. They are the only food I stopped eating as a result of my EIS experience."

I finished Inside the Outbreaks while traveling, and as I looked for a snack in the air- port, I had difficulty identifying food that would be safe. The yogurt looked terrific, but it contained berries, and I had just read about outbreaks of infections in Texas and Florida with the parasite Cyclospora, caused by raspberries from Guatemala. There were also lovely sandwiches, but who knew what lurked in the salad greens - perhaps E. coli O157:H7, which caused gastroenteritis in Illinois when it contaminated mesclun from California. Reading Inside the Outbreaks will cause you to suspect nearly every food or food supplement, as well you should. The global economy and the demand for fresh food throughout the year have led to many opportunities for traveling microbes.

The list of former EIS officers is a Who's Who of significant figures in science and medicine. Some individuals I was surprised to find in this program include D.A. Henderson and William Foege, architects of the smallpox eradication program; Neal Nathanson, a prominent virologist; current CDC Director Tom Frieden; former CDC Director Julie Gerberding; and WHO Assistant Director-General Keiji Fukuda. Neither had I known that Lawrence Altman, the wellknown New York Times science writer, had been an EIS officer.

You'll have to read Inside the Outbreaks to learn how an EIS trainee learns the craft of disease epidemiology. Perhaps Alexander Langmuir's approach is the most informative: he would send only one or two EIS officers to an outbreak. "We'll get them on an epidemic as fast as we can. Throw them overboard. See if they can swim, and if they can't, throw them a life ring; pull them out and throw them in again." 